\title{
Salivary Interleukin-1ß Levels in Chronic Periodontitis Patients after use of Nigella Sativa (Kalonji) Oil
}

\author{
Ghazala Hassan $^{1}$ \\ Sarah Ghafoor ${ }^{2}$ \\ Saima Chaudhry ${ }^{3}$ \\ Zubair Ahmed Khan ${ }^{4}$
}

\author{
BDS \\ BDS, B.Sc, MPHE, PhD \\ BDS, B.Sc, MME, PhD \\ BDS, MCPS
}

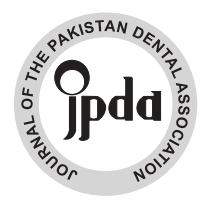

OBJECTIVE: Periodontitis is the second most prevalent microbiome associated inflammatory disease posing a threat to oral health. Nigella sativa (Kalonji) has been used since ancient times as a remedy for oral inflammatory conditions. Interleukin1 (IL-1 ) is critical for periodontal inflammation, collagen degradation and bone turn over. The motive of our study was to determine the change in the levels of salivary IL-1 after the use of Nigella sativa oil to determine if it has any correlation with salivary IL-1 .

METHODOLOGY: A parallel-arm triple-blind placebo-based randomized control trial was conducted on a total of ninety three patients. Out of these, fifty individuals with chronic periodontitis were included in the study as per the eligibility criteria. Baseline screening of the participants was done via clinical periodontal parameters such as periodontal pocket depth (PPD), clinical attachment loss (CAL), plaque index (PI) and bleeding on probing (BoP). These individuals were categorized into two groups; 1. Control Group ( $\mathrm{n}=25)$, which was given normal saline as placebo; 2. Treatment Group, which was given Nigella sativa oil $(\mathrm{n}=25)$. All participants underwent scaling and root planing before the start of the trial. The intervention was given for two weeks. Salivary samples were collected on day 0 and day 15 and were evaluated for interleukin-1 levels using ELISA. The statistical interpretation was done using IBM SPSS (version 25.0, SPSS Inc.) on forty participants due to loss to follow up.

RESULTS: Levels of salivary interleukin-1 came out to be statistically insignificant after two-week use of Nigella sativa oil. CONCLUSION: No correlation was found between the salivary IL-1 and the use of Nigella sativa oil in patients with chronic periodontitis in contrast to the patients using normal saline.

KEYWORDS: Chronic periodontitis, Interleukin-1 , Nigella sativa, Kalonji, Salivary

HOW TO CITE: Hassan G, Ghafoor S, Chaudhry S, Khan ZA. Salivary interleukin-1 Levels in chronic periodontitis patients after use of nigella sativa (Kalonji) oil. J Pak Dent Assoc 2020;29(4):205-210.

DOI: https://doi.org/10.25301/JPDA.294.205

Received: 13 April 2020, Accepted: 28 August 2020

\section{INTRODUCTION}

$\mathrm{P}$ eriodontitis is a chronic, inflammatory disease that poses an oral health challenge in human populations around the world. Almost 20-50\% of the world's population suffers from periodontitis having different forms including "chronic periodontitis" (CP). ${ }^{1}$ This chronic inflammatory condition is initiated by bacteria present in

1. M. Phil Trainee-II, Department of Oral Biology, University of Health Sciences, Khayaban-e-Jamia, Lahore, Punjab.

2. Assistant Professor, Department of Oral Biology, University of Health Sciences, Khayaban-e-Jamia, Lahore, Punjab.

3. Associate Professor, Department of Oral Pathology, University of Health Sciences, Khayaban-e-Jamia, Lahore, Punjab.

4. Associate Professor, Department of Periodontology, Fatima Memorial College of Medicine and Dentistry, Shadman, Lahore.

Corresponding author: "Dr. Sarah Ghafoor" < sarahghafoor@uhs.edu.pk > plaque and if untreated it leads to calculus formation. The disruption of the normal oral microflora due to plaque accumaulation initiates a chronic, inflammatory response in the oral tissues. These events activates a variety of immune responses that lead to pathological process and progression of chronic periodontitis involving gingival inflammation and bleeding, gradual loss of clinical attachment, and bone loss. Periodontal treatment mainly involves root surface debridement of dental plaque or calculus to reduce the microbial load followed by advice on regular oral hygiene measures to the patient. ${ }^{2,3}$ A number of inflammatory cytokines such as tumor necrosis factor- alpha (TNF- ), interleukin-6 (IL-6), interleukin-1 (IL-1 ), interleukin-12 (IL-12), interleukin-17 (IL-17), interleukin-18 (IL-18) are linked to oral infections such as oral lichen planus (OLP), vesicobullous diseases and other systemic diseases like 
diabetes, chronic heart disease, etc. ${ }^{4,5}$ Moreover, -glucuronidase, C-reactive protein, IL-1, IL-6, TNF- and MMP- 8 have been specifically used biomarkers for chronic periodontitis in the past. ${ }^{6}$ Among these, interleukin-1 (IL-1 ) is considered an important indicator of chronic periodontitis and has been effectively diagnosed in saliva. ${ }^{7}$

Saliva, being a unique biological fluid as it contains many proteins and enzymes that can be used as biomarkers for early detection of oral diseases and evaluation of therapeutic response. ${ }^{4,8}$ Thus it provides a rapid and noninvasive diagnostic tool for determining disease conditions. Saliva has been effectively used for the detection of inflammatory biomarkers such as IL-1 , IL-6, IL-8, TNF- , lysozyme, MMP-8 for diseases like oral lichen planus, oral cancers, vesicobullous diseases and periodontitis. ${ }^{8,9}$

Apart from conventional treatment modalities by the dentists for chronic periodontal disease, a wide array of active ingredients present in naturallay occuring medicinal herbs have been used for its cure. Nigella sativa (NS) commonly known as "Kalonji" in Urdu language, is a herb that has therapeutic potential for cure of oro-dental diseases. ${ }^{10}$ Its active ingredient thymoquinone (TQ) is well studied for anti-inflammatory role in many systemic diseases such as asthma, diabetes mellitus, hypercholestremia, cardiovascular disorders and autoimmune disease. ${ }^{11}$ Previous studies suggest that NS has effect on reducing symptoms of chronic periodontitis clinically by reducung the plaque index, gingival swelling and improving the clinical attachment loss. ${ }^{12}$ Other studies have also suggested its role at molecular level by decreasing the levels of inflammatory biomarkers such as IL-1 and MMP-8 in systemic conditions like hypercholestremia, and arthritis yet only fewer studies are available on effects of NS on chronic periodontitis in humans. ${ }^{12,13}$ It is favourable to use Nigella sativa oil for periodontal problems due to its cost-effectiveness and fewer side effects as compared to chlorhexidine mouthwash that is generally used after chronic periodiodontitis therapy for oral hygiene, making it a better adjuvant for periodontal treatment. ${ }^{14}$ Studies suggest, that Nigella sativa oil leads to changes in the levels of IL-1 in various inflammatory conditions such as rheumatoid arthritis, asthma, diabetes mellitus, etc. ${ }^{15,16}$ The objective of the study was to understand the role of Nigella sativa oil in chronic periodontitis disease which was assessed through the evaluation of levels of salivary IL-1 in chronic periodontitis patients.

\section{METHODOLOGY}

The study was done following the CONSORT 2010 guidelines. This study was a parallel-arm triple-blind placebo- based randomized clinical trial (Trial Registration. No. NCT03270280) that was carried out in the Oral Biology Department, University of Health Sciences, Lahore and Dental Diagnostic and Periodontology Department of Fatima Memorial Hospital, Shadman, Lahore (Figure 1). An ethical approval certificate was obtained from the Ethical Review Committee of the University of Health Sciences, Lahore and Institutional Review Board of Fatima Memorial College of Medicine and Dentistry (IRB \# FMH-07-2017-IRB-268F) before the commencement of the study.

A total of ninety three participants were screened for chronic periodontitis out of which fifty chronic periodontitis patients were included in the study as per the eligibility criteria. Participants who were smokers and those who had systemic illnesses, allergies, pregnancy, any drug intake and periodontal treatment in the past six months were excluded from the study. All the patients signed a detailed consent form before the initiation of the clinical trial. Unstimulated saliva was collected in a graded tube through passive drooling technique in the morning (between 08:00-09:00 a.m.) and the participants were instructed to refrain from eating and drinking and was not allowed to perform oral hygiene measures for at least 1 hour before to the saliva collection. ${ }^{17}$ The participants were asked to rinse their mouth out with distilled drinking water for at least 1 minute, and then they could either swallow it or expectorate. Sterilized cotton pellets were used to remove any food debris. After the oral rinse, the participants were asked to drool saliva passively into a $50 \mathrm{ml}$ sterile tube. The tube was placed on ice while more saliva was collected from the subject. Approximately $5 \mathrm{ml}$ of saliva was collected. Collected samples were placed on ice at once and transferred to $2.5 \mathrm{ml}$ aliquots in eppendorf tubes before storing at $-80 \mathrm{C} .{ }^{18}$

These participants were divided into two groups comprising twenty five participants in each group (Figure 1). Screening of patients was done via baseline clinical parameters such as probing pocket depth (PPD), clinical attachment loss (CAL), plaque index (PI) and bleeding on probing (BOP) were measured using the periodontal chart. ${ }^{12}$ Scaling and root planing was performed on each study participants before the start of the clinical trial. Salivary samples were taken on day 0 and day 15 from all the patients. The control group and treatment group were given normal saline (UNISOL-NS®, Pakistan) or Nigella sativa oil (Kalonji oil-Marbaha ${ }^{\circledR}$ ) in amber bottles, respectively, to use topically on the gingiva twice daily for two weeks. Each patient received $80 \mathrm{ml}$ of the mouthwash and was instructed to apply 55 drops $(5 \mathrm{ml})$ of it on gingiva.

\section{Randomization and Blinding of the Participants}

Fifty filled amber bottles with half of them containing NS 
oil and half containing normal saline were randomly allocated numerical numbers starting from one till fifty through a random key generator. The allocated numbers were labelled on amber bottles. The researcher was unaware of the contents of the bottle as the allocation was done by a third person. The researcher handed the bottles to each study participant after the initial scaling and root planing procedure. Similarly the patients who received either NS oil or normal saline were also kept blinded regarding the contents of the labelled bottles. Later, when the data was compiled, the statistician was also kept unaware of the allocation of the bottles to the study participants. Thus, triple blinding was ensured to avoid selection bias in the study.

\section{Quantitative Analysis of Salivary Interleukin-1及}

Quantitative detection of salivary interleukin-1 was carried out using ELISA through a salivary IL-1 kit (SinoGeneClon Biotech Co., Ltd SG-10260) in Oral Biology laboratory, University of Health Sciences, Lahore, Pakistan. Dilution of the standard solutions in the kit was done as per the manufacturer's guidelines. The salivary samples were pipetted to each well pre-coated with IL-1 specific antibodies. ${ }^{19}$ All assay procedures were carried out following the manufacturer's specifications. ${ }^{20}$ The reaction was stopped using a stop solution, and the optical density (O.D) values were determined by a spectrophotometric ELISA-Reader (Biochrom EZ read 400) at a wavelength of $450 \mathrm{~nm}$ using Galapagos $^{\mathrm{TM}}$ software.

\section{STATISTICAL ANALYSIS}

Statistical analysis was carried out using IBM SPSS (Version 25.0) on forty participants due to the drop-out of ten participants in both groups. Since the data of salivary marker IL-1 was found to not be normally distributed, nonparametric test i-e- Mann-Whitney-U test was used for the comparison of IL-1 concentrations in salivary samples between the treatment group and control group. The pretreatment and post-treatment salivary IL-1 levels in each group individually were analyzed using a paired t-test. The p-value of $\leq 0.05$ is considered to be significant. ${ }^{21}$

\section{RESULTS}

According to the demographic data collected, the mean age of the study participants came out to be thirty six years, and there were eleven males and nine females in both the groups. Salivary samples of forty chronic periodontitis were evaluated for the comparison of concentration and optical density (OD) values of salivary IL-1 through ELISA after scaling and root planing and after two weeks' use of either
Nigella sativa oil or normal saline (Table 1). The results revealed that the control group had slightly lower post-treatment mean optical density (O.D) had $0.11 \pm 0.049$ value than the pre-treatment mean O.D had $0.12 \pm 0.037$ value. A paired t-test showed this difference to be statistically

Table 1: Baseline characteristics of study participants

\begin{tabular}{|c|c|c|}
\hline \multicolumn{3}{|c|}{ First Visit (Baseline) } \\
& 40 Chronic Periodontitis Patients \\
\hline \multirow{2}{*}{ Age (Years) } & Control Group & Treatment Group \\
\hline Male & $38.27 \pm 7.44$ & $36.39 \pm 8.14$ \\
\hline Female & $11(55 \%)$ & $11(55 \%)$ \\
\hline Interleukin-1 $\beta \mathrm{ng} / \mathrm{L}$ & $\mathbf{9 ( 4 5 \% )}$ & $\mathbf{9 ( 4 5 \% )}$ \\
\hline
\end{tabular}

Table 2: Analysis of Salivary Interleukin 1 Levels in Control and Treatment Groups

\begin{tabular}{|c|c|c|c|c|c|c|c|}
\hline \multicolumn{8}{|c|}{ Salivary of IL-1 $1 \beta$ between Optical Density and Concentration } \\
\hline \multicolumn{8}{|c|}{ Between Control and Treatment Group } \\
\hline & \multicolumn{4}{|c|}{ Control Group } & $\begin{array}{c}\text { Treatment } \\
\text { Group }\end{array}$ & \multicolumn{2}{|c|}{$p$-value } \\
\hline \multirow[b]{2}{*}{$\begin{array}{l}\text { Optical } \\
\text { Density }\end{array}$} & \multicolumn{2}{|c|}{ Pre-treatment } & \multicolumn{2}{|c|}{$0.12 \pm 0.03$} & $0.11 \pm 0.04$ & \multicolumn{2}{|c|}{1.68} \\
\hline & \multicolumn{2}{|c|}{ Post-treatment } & \multicolumn{2}{|c|}{$0.11 \pm 0.049$} & $0.12 \pm 0.059$ & \multicolumn{2}{|c|}{0.665} \\
\hline \multirow[b]{2}{*}{ Concentration } & & \multicolumn{2}{|c|}{$5.46 \pm 2.06$} & $4.47 \pm 2.34$ & \multicolumn{2}{|c|}{1.68} \\
\hline & & Post-treatment & \multicolumn{2}{|c|}{$5.17 \pm 2.76$} & $5.80 \pm 3.37$ & \multicolumn{2}{|c|}{0.665} \\
\hline \multicolumn{8}{|c|}{ Within Control and Treatment Group } \\
\hline & \multicolumn{4}{|c|}{ Control Group } & \multicolumn{3}{|c|}{ Treatment Group } \\
\hline & $\begin{array}{c}\text { Pre- } \\
\text { Treatment }\end{array}$ & & $\begin{array}{l}\text { st- } \\
\text { ment }\end{array}$ & $p$-value & Pre-treatment & $\begin{array}{c}\text { Post- } \\
\text { treatment }\end{array}$ & $p$-value \\
\hline Optical Density & $0.12 \pm 0.03$ & & 0.04 & 0.75 & $0.11 \pm 0.04$ & $0.12 \pm 0.05$ & 0.09 \\
\hline Concentration & $5.46 \pm 2.06$ & & & 0.78 & $4.47 \pm 2.34$ & $5.81 \pm 3.37$ & 0.09 \\
\hline
\end{tabular}

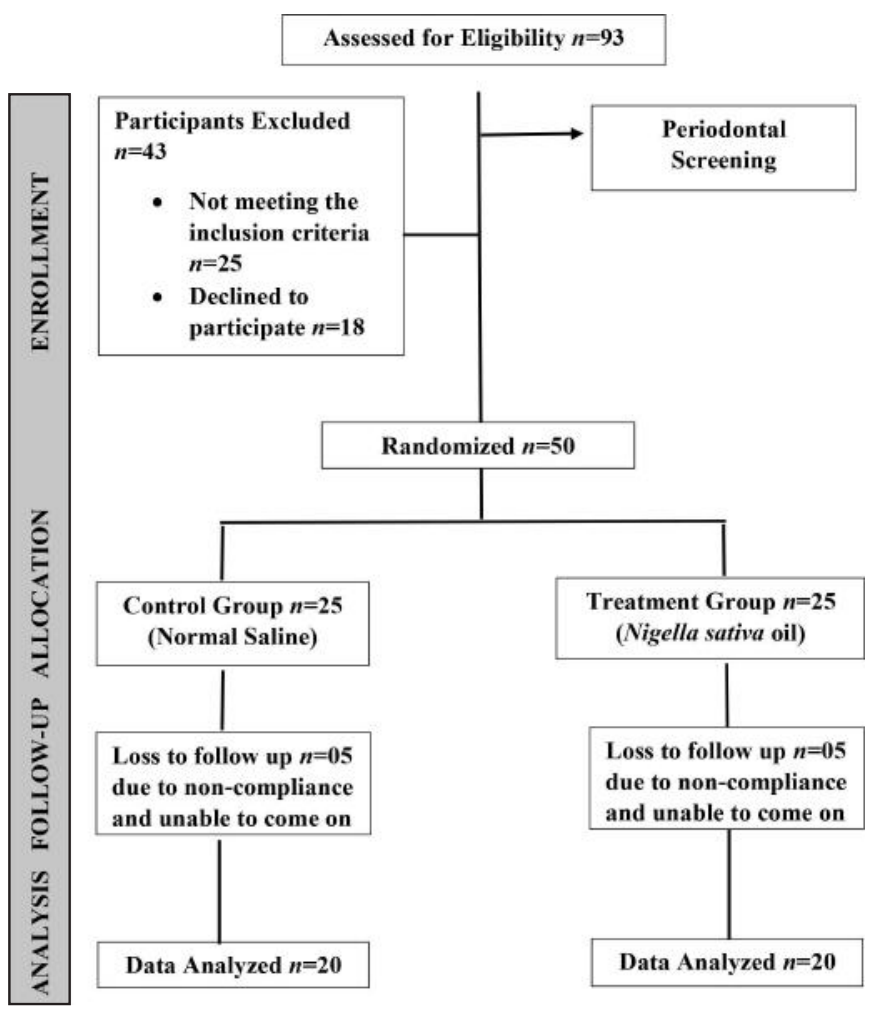


insignificant as the p-value came out to be 0.75 . The results for pre-treatment and post-treatment concentration values of the control group came out to be $5.46 \pm 2.06$ and $5.17 \pm 2.76$ with a p-value of 0.786 . The paired t-test showed that the results were statistically insignificant, which suggests that no changed had occurred in the levels of salivary IL-1 in the control group after the use of normal saline (Table 2). The mean \pm SD for O.D values of treatment group for pre-treatment and post-treatment analysis was almost similar to an insignificant $p$-value of 0.094 . The difference between the mean pre-treatment and post-treatment concentrations of treatment group $4.47 \pm 2.34$ and $5.81 \pm 3.37$, respectively showed irrelevant results as the p-value was 0.093 . These results suggest that there was no change found in the levels of salivary IL-1 in the treatment group after using Nigella sativa oil (Table 2).

\section{DISCUSSION}

Besides caries, $\mathrm{CP}$ is one of the most common oral diseases that is caused by the inflammation of the periodontium of the tooth. The progression of the disease from gingivitis to periodontitis depends on the degree of inflammation. But the exact mechanism is poorly understood. The sequel of periodontal tissue destruction in $\mathrm{CP}$ initiates with the deposition of dental plaque. ${ }^{22}$ Plaque pathogens keeps on accumulating until they are removed either by oral hygiene measures or standard periodontal therapy i.e. scaling and root planing. If these standard procedures are not ensured, the periodontal disease will progress and will result in an on-going inflammatory response creating a set of biological markers such as TNF- , IL-1 , IL-6 and tissue destructive elements. ${ }^{23,24}$ Elevated levels of MMP-8 (neutrophil collagenase), MMP-9 (neutrophil gelatinase), and -glucuronidase are reflected in the periodontium due to enhanced local activity of the neutrophils. ${ }^{25}$ If this condition left untreated, it would lead to tissue destruction, which is the hallmark of chronic periodontitis, eventually leading to the loss of teeth. ${ }^{26}$

We found the comparison of salivary IL-1 levels in chronic periodontitis patients remained unchanged after the use of Nigella sativa oil as compared to normal saline. According to our results, the comparison of salivary IL-1 concentration between control and treatment group came out to be statistically insignificant. Even when we compared the levels of IL-1 within the same group before and after the treatment with NS oil, the results indicated statistically insignificant values. Although, not directly related, but our results in contrast findings in some of the previously done research that suggest that levels of IL-1 are reduced in inflammatory conditions such as pancreatic cell cancer and rheumatoid arthritis after the use of Nigella sativa. ${ }^{27,28}$

However, previous studies show that IL-1 is a robust inflammatory biomarker for chronic periodontitis. ${ }^{24,29}$ Previously, a study showed an increase in the levels of salivary IL-1 in CP patients as compared to individuals without CP. ${ }^{30}$ Another study found that the levels of IL-1 were notably higher in individuals with periodontitis than gingivitis and healthy patients. ${ }^{31} \mathrm{IL}-1$ levels increase as the periodontal disease progresses through its various stages and also in different types of periodontal diseases. ${ }^{19}$

Nigella sativa has effective anti-plaque, anti-bacterial, anti-inflammatory and anti-oxidant properties. In 2014, Al-Wafi et al studied the potential inhibitory role of thymoquinone (TQ) on gingival inflammation in rat models. The results showed that TQ effectively diminished plaque formation. ${ }^{13}$ Most of the studies that were conducted to investigate the role and effectiveness of Nigella sativa in periodontitis utilized rat models. These studies reported a decrease in the levels of inflammatory markers such as IL-1 , TNF- and MMP-8 in rat models owing to a special ingredient of Nigella sativa i-e- thymoquinone. ${ }^{27,28,32}$ However, we did not find any change in levels of IL-1 in human samples. Thus, there is a possibility that if we would have increased the duration of the treatment, we may have achieved different results. This view can be strengthened by a recently done study that concluded that the biochemical changes as a result of periodontitis could be reversed at least after 4 weeks use of thymoquinone (an active ingredient of Nigella sativa). ${ }^{33}$

\section{CONCLUSION}

No change was observed in salivary IL-1 before and after treatment with Nigella sativa oil in chronic periodontitis patients following scaling and root planning in our study, however, NS is still an effective alternative herbal option available for treatment of many dental diseases.

\section{LIMITATION OF THE STUDY}

The duration of the study for the usage of Nigella sativa (Kalonji oil) was only two weeks and therefore, results cannot be generalized for similar outcomes in a longer duration study.

\section{ACKNOWLEDGMENTS}

The authors will like to thank all the study participants who volunteered in this study. We are also thankful to the Higher Education Commission of Pakistan (HEC) for providing e-Library access to the University of Health 
Sciences Lahore, without which published data could not be accessed.

\section{CONFLICT OF INTEREST}

None declared.

\section{REFERENCES}

1. Nazir MA. Prevalence of periodontal disease, its association with systemic diseases and prevention. Int J Health Sc. 2017;11:72.

2. Darby M, Walsh M. Dental Hygiene Theory and Practice. 3rd. St Louis, Mo, USA: Saunders Elsevier. 2010.

3. Lauritano D, Bignozzi CA, Pazzi D, Palmieri A, Gaudio RM, Di Muzio M, et al. Evaluation of the efficacy of a new oral gel as an adjunct to home oral hygiene in the management of chronic periodontitis. A microbiological study using PCR analysis.J Biol Reg Homeos Ag. 2016;30:123-28.

4. Nasim H, Ghafoor S. Proteins Regulating Salivary and Lacrimal Flow in Xerostomia and Dry Eye Syndrome. J Pak Dent Assoc. 2019;28:93.

https://doi.org/10.25301/JPDA.282.92

5. Rathnayake N, Åkerman S, Klinge B, Lundegren N, Jansson H, Tryselius Y, et al. Salivary biomarkers for detection of systemic diseases. PloS one. 2013;8:e61356.

https://doi.org/10.1371/journal.pone.0061356

6. Rangbulla V, Nirola A, Gupta M, Batra P, Gupta M. SalivaryIgA, Interleukin-1beta and MMP-8 as salivary biomarkers in chronic periodontitis patients. Chin J Dent Res. 2017;20:43-51.

7. Cheng R, Wu Z, Li M, Shao M, Hu T. Interleukin-1ß is a potential therapeutic target for periodontitis: a narrative review. Int J Oral Sci. 2020;12:1-9.

https://doi.org/10.1038/s41368-019-0068-8

8. Martina E, Campanati A, Diotallevi F, Offidani A. Saliva Oral Dis. J Clin Med. 2020;9:466.

https://doi.org/10.3390/jcm9020466

9. Buzalaf MAR, Ortiz AdC, Carvalho TS, Fideles SOM, Araújo TT, Moraes SM, et al. Saliva as a diagnostic tool for dental caries, periodontal disease and cancer: is there a need for more biomarkers? Expert Rev. Mol. Diagn. 2020;20:543-55.

https://doi.org/10.1080/14737159.2020.1743686

10. AlAttas SA, Fat'heya MZ, Turkistany SA. Nigella sativa and its active constituent thymoquinone in oral health. Saudi Med J. 2016;37:235.

https://doi.org/10.15537/smj.2016.3.13006

11. Hallajzadeh J, Milajerdi A, Mobini M, Amirani E, Azizi S, Nikkhah E, et al. Effects of Nigella sativa on glycemic control, lipid profiles, and biomarkers of inflammatory and oxidative stress: A systematic review and meta-analysis of randomized controlled clinical trials. Phytother Res. 2020;34:2586-08. https://doi.org/10.1002/ptr.6708

12. Al-Bayaty FH, Kamaruddin AA, Ismail M, Abdulla MA. Formulation and evaluation of a new biodegradable periodontal chip containing thymoquinone in a chitosan base for the management of chronic periodontitis. J Nanomater. 2013;Article ID 397308.

https://doi.org/10.1186/ISRCTN29742423

13. Al-Wafi HA. Benefits of Thymoquinone, a Nigella Sativa Extract in Preventing Dental Caries Initiation and Improving Gingival Health: (Doctoral dissertation, Tufts University School of Dental Medicine); 2014.

14. Hassan G, Ghafoor S. Herbal Medicines: An Adjunct to Current Treatment Modalities for Periodontal Diseases. Biomedica. 2020;36.

15. Mohit M, Farrokhzad A, Faraji SN, Heidarzadeh-Esfahani N, Kafeshani M. Effect of Nigella sativa L.Supplementation on inflammatory and oxidative stress indicators: A systematic review and meta-analysis of controlled clinical trials. Complement Ther Med. 2020:102535.

https://doi.org/10.1016/j.ctim.2020.102535

16. Sandhya A, Kannayiram G. Pharmacological, bioactive screening of medicinal plant Nigella sativa and the derived compound thymoquinone: An invitro study. Int J Res Pharmaceut Sc. 2020;11:245865.

https://doi.org/10.26452/ijrps.v11i2.2239

17. Salimetrics L, SalivaBio L. Saliva collection and handling advice. State College, PA: Salimetrics LLC, SalivaBio LLC. 2011:1-14.

18. Henson BS, Wong DT. Collection, storage, and processing of saliva samples for downstream molecular applications. Oral Biol: Springer; 2010. p. 21-30.

https://doi.org/10.1007/978-1-60761-820-1_2

19. Ertugrul A, Sahin H, Dikilitas A, Alpaslan N, Bozoglan A. Comparison of CCL28, interleukin-8, interleukin-1ß and tumor necrosis factor-alpha in subjects with gingivitis, chronic periodontitis and generalized aggressive periodontitis. J Periodontal Res2013;48:4451.

https://doi.org/10.1111/j.1600-0765.2012.01500.x

20. Seymour GJ, Cullinan MP, Heng NC. Oral biology: molecular techniques and applications: Springer; 2010.

https://doi.org/10.1007/978-1-60761-820-1

21. D'Aiuto F, Parkar M, Nibali L, Suvan J, Lessem J, Tonetti MS. Periodontal infections cause changes in traditional and novel cardiovascular risk factors: results from a randomized controlled clinical trial. Am Heart J. 2006;151:977-84.

https://doi.org/10.1016/j.ahj.2005.06.018

22. Mauramo M, Ramseier A, Mauramo E, Buser A, Tervahartiala T, Sorsa T, et al. Associations of oral fluid MMP-8 with periodontitis in Swiss adult subjects. Oral Dis. 2018;24:449-55.

https://doi.org/10.1111/odi.12769 
23. Kinane DF, Preshaw PM, Loos BG, Periodontology WGotSEWo. Host-response: understanding the cellular and molecular mechanisms of host-microbial interactions-Consensus of the Seventh European Workshop on Periodontology. J Clin Periodontol. 2011;38:44-8. https://doi.org/10.1111/j.1600-051X.2010.01682.x

24. Rathnayake N, Åkerman S, Klinge B, Lundegren N, Jansson H, Tryselius Y, et al. Salivary biomarkers of oral health-a cross-sectional study. J Clin Periodontol. 2013;40:140-7.

https://doi.org/10.1111/jcpe.12038

25. Sorsa T, Gursoy UK, Nwhator S, Hernandez M, Tervahartiala T, Leppilahti J, et al. Analysis of matrix metalloproteinases, especially MMP-8, in gingival crevicular fluid, mouthrinse and saliva for monitoring periodontal diseases. Periodontol 2000. 2016;70:142-63. https://doi.org/10.1111/prd.12101

26. Taylor JJ. Protein biomarkers of periodontitis in saliva. ISRN inflammation. 2014;2014.

https://doi.org/10.1155/2014/593151

27. Chehl N, Chipitsyna G, Gong Q, Yeo CJ, Arafat HA. Antiinflammatory effects of the Nigella sativa seed extract, thymoquinone, in pancreatic cancer cells. HPB. 2009;11:373-81.

https://doi.org/10.1111/j.1477-2574.2009.00059.x

28. Tekeoglu I, Dogan A, Ediz L, Budancamanak M, Demirel A. Effects of thymoquinone (volatile oil of black cumin) on rheumatoid arthritis in rat models. Phytoth Res: An Int J Devot Pharma Toxicol Eval Nat Prod Derivat 2007;21:895-97.

https://doi.org/10.1002/ptr.2143

29. Kinney J, Morelli T, Braun T, Ramseier CA, Herr A, Sugai J, et al. Saliva/pathogen biomarker signatures and periodontal disease progression. J Dent Res. 2011;90:752-8.

https://doi.org/10.1177/0022034511399908

30. Stashenko P, Fujiyoshi P, Obernesser M, Prostak L, Haffajee A, Socransky S. Levels of interleukin $1 B$ in tissue from sites of active periodontal disease. J Clin Periodontol. 1991;18:548-54.

https://doi.org/10.1111/j.1600-051X.1991.tb00088.x

31. Faizuddin M, Bharathi S, Rohini N. Estimation of interleukin-1ß levels in the gingival crevicular fluid in health and in inflammatory periodontal disease. J Periodontal Res. 2003;38:111-4.

https://doi.org/10.1034/j.1600-0765.2003.01649.x

32. Ozdemir H, Kara M, Erciyas K, Ozer H, Ay S. Preventive effects of thymoquinone in a rat periodontitis model: a morphometric and histopathological study. J Periodontal Res. 2012;47:74-80.

https://doi.org/10.1111/j.1600-0765.2011.01406.x

33. Alaaeldin E, Gomaa MD, Eltewacy NK, Ali A, Khalil AA. Formulation and Characterization of Thymoquinone Bioadhesive Gel for Treatment of Chronic Gum Inflammation. Int J Sci (Basic and Applied Research) 2017;31:321-30 